\title{
Propuesta de itinerario interdisciplinar en la formación del profesorado de Educación Primaria en el ámbito de las Ciencias Sociales y Experimentales
}

\section{Proposed itinerary interdisciplinary teacher training of primary education in the field of Social Sciences and Experimental}

\section{Silvia Medina Quintana, José Carlos Arrebola Haro, Manuel Mora Márquez, José Antonio López Fernández}

Dpto. de Didáctica de las Ciencias Sociales y Experimentales. Universidad de Córdoba

\begin{abstract}
Resumen: Se propone en este trabajo un itinerario didáctico con un planteamiento interdisciplinar en la formación del profesorado de Educación Primaria cuya experiencia se está llevando a cabo en la Facultad de Ciencias de la Educación de la Universidad de Córdoba, entre las áreas de Didáctica de las Ciencias Sociales y Experimentales, a través de una metodología activa. El alumnado es el que lleva a cabo la preparación de las paradas y la exposición de las mismas. Esta propuesta de colaboración entre estas dos áreas fija sus objetivos en la participación integral a través de la búsqueda y análisis de información relevante, el trabajo colaborativo, así como la presentación de los resultados al resto del alumnado. La tarea fomenta capacidades y competencias relacionadas con la transversalidad y la interdisciplinariedad de los contenidos en Educación Primaria.
\end{abstract}

Palabras clave: Didáctica de las Ciencias Sociales y Experimentales; Itinerario didáctico; Transversalidad; Interdisciplinariedad.

Abstract: The aim of this work is to develop an educational itinerary to describe an interdisciplinary proposal that it is being developed in the Bachelor Degree of Primary Education belonging to the Faculty of Education Sciences in the University of Córdoba, with the implication of two areas of knowledge: Didactics of Social Sciences and Didactics of Experimental Sciences. We implemented an educational itinerary through the city of Cordoba, in which the students had to prepare and explain several aspects of the different points of interest in the itinerary, regarding with Social and/or Experimental Sciences. This activity implies an active and participative methodology that allows the students to develop some competencies as cooperative work, searching for information or oral presentations. Furthermore, this also promotes both interdisciplinarity and transversality. The main objective of this activity is to make the students reflect on the importance of their surroundings and on the possibilities that Cordoba city offers to be used as an educational resource.

Keywords: Didactics of Social and Experimental Sciences; educational itinerary; transversality; interdisciplinarity.

(Fecha de recepción: febrero, 2016, y de aceptación: septiembre, 2016)

DOI: 10.7203/DCES.31.8058 


\section{Introducción}

El espacio que rodea al alumnado puede abordarse, desde el ámbito educativo, a través de numerosas materias. No en vano, casi todos los acontecimientos sociales se producen en uno o varios lugares. El hecho de abordar el espacio puede realizarse desde edades tempranas mediante la Educación Física, con la realización de ejercicios de orientación, coordinación, etc.; en las Ciencias de la naturaleza, ya que todos los procesos ocurren en determinadas condiciones ecológicas y, por lo tanto, espaciales. $\mathrm{Y}$, sobre todo, a través de las Ciencias Sociales, donde el ser humano utiliza el espacio y acondiciona su propio teatro de actuación (el territorio). Por ello, se considera que las diferentes materias que están relacionadas con el espacio deben utilizar éste para enseñar al estudiante los diferentes procesos que ocurren en él, para que lo viva, lo comprenda, lo racionalice y lo localice; y, principalmente, para que entienda las diferentes relaciones que tienen lugar en él.

Este trabajo pretende mostrar el proyecto en desarrollo que se lleva a cabo en el tercer curso del Grado de Educación Primaria de la Facultad de Ciencias de la Educación de la Universidad de Córdoba (España), planificado por las áreas específicas de Didáctica de Ciencias Sociales y Didáctica de Ciencias Experimentales; a través de una metodología activa planteada sobre un mismo itinerario base, donde el profesorado ha jugado el papel de guía, a la que vez que ha tutorizado a los diferentes grupos.

Entre los principales objetivos del trabajo destaca el desarrollo competencial del trabajo colaborativo entre el alumnado, la búsqueda y selección de información, así como su preparación para exponerla de forma adecuada al resto del grupo durante el desarrollo del itinerario. Asimismo, el alumnado trabaja diferentes aspectos relacionados con las materias de conocimiento, desarrolla capacidades y competencias de índole espacial, así como tratar cuestiones de carácter local, hasta ahora desconocidos, como elementos de la cultura y el patrimonio. Los objetivos didácticos más específicos se orientan hacia la utilidad del espacio, en este caso urbano, como recurso (donde se pueden tratar diferentes aspectos sociales, culturales, naturales, patrimoniales, etc.), gracias a un planteamiento metodológico donde los discentes participan en la preparación y desarrollo de la actividad, sin permanecer como meros espectadores.

Esta actividad fomenta la coordinación entre las dos áreas señaladas que, aunque se engloban en un departamento específico e intervienen de forma conjunta en varias asignaturas, gracias a la realización de este proyecto se favorece y promueve el trabajo colaborativo del personal docente universitario, lo que ha propiciado unos objetivos comunes. Por ello, la implementación de este trabajo permite comprobar a los estudiantes la importancia del trabajo en equipo, tanto del profesorado universitario como entre ellos mismos. 


\section{El trabajo fuera del aula}

\section{Salir fuera del aula}

Hace unos años, la revista Iber dedicaba un monográfico a la escuela y el aprendizaje fuera del aula, dirigido por Araceli Vilarrasa (2003) y titulado, precisamente, "Salir del aula". Esta autora señalaba una serie de reflexiones que continúan vigentes a la hora de planificar una actividad escolar que tiene lugar en un escenario diferente al cotidiano. El objetivo de estas salidas no debe ser nunca el mero divertimento, sino "la posibilidad de llevar la escuela a todos aquellos espacios en los que se hace posible la vivencia de experiencias culturales sobre las que construir el conocimiento y acercar el aprendizaje disciplinar a la experiencia" (2003a, p. 5).

La necesidad de realizar un aprendizaje activo fuera de las aulas se sigue reclamando en la actualidad, pero ya fue puesta de manifiesto en el pasado por diversas corrientes pedagógicas renovadoras. Así, por ejemplo, destacan Pestalozzi quien, entre el siglo XVIII y XIX, ya defendía la importancia de que los y las estudiantes estuviesen en contacto con la naturaleza y de que su aprendizaje partiera de su propia experiencia. Discípulo de Pestalozzi fue un destacado pedagogo, Friedrich Froëbel, quien defendía igualmente las salidas fuera del aula y del aprendizaje a través del juego. Metodologías lúdicas que han llevado a cabo otros autores más recientes, para muchos contenidos relacionados con las Ciencias Sociales en general y la Geografía en particular (Marrón, 2005 y 2012).

A finales del siglo XIX y durante el siglo XX se desarrolla la corriente renovadora de la Escuela Nueva, con nombres como John Dewey, Jean Piaget, Maria Montessori y Célestin Freinet, entre otros. Esta forma de entender la enseñanza promovía la necesidad de salir fuera del aula como parte del aprendizaje del alumnado basado en la experiencia (Vilarrasa, 2003b, p. 14). En España, la Institución Libre de Enseñanza (ILE), que se movía en las directrices de la Escuela Nueva, supuso también un punto de inflexión en la forma tradicional de abordar la escolarización. Como ejemplo podemos citar un texto de Pablo Miaja en el Boletín del Instituto Libre de Enseñanza, de 1919, donde se valora positivamente la inclusión del entorno en el aprendizaje infantil. Este autor aboga por combinar lecciones teóricas con salidas exteriores para afianzar los conocimientos adquiridos: "Las excursiones escolares vienen a combatir enérgicamente el error capitalísimo de la enseñanza en la escuela, cual es el prescindir lastimosamente de la educación, dándolo todo a la instrucción. Grave cáncer cuya extirpación se impone, si queremos entonar el himno del triunfo" (Ruiz y Palacio, 1999, p. 179). Otros autores vinculados también a la ILE, como Rafael Altamira, Pau Vila y Francisco Ferrer Guardia, defendieron igualmente la necesidad de acercarse al entorno inmediato y trabajar los contenidos escolares a través del medio (Hernández, 2002, p. 270-271). 
A pesar de esta tradición pedagógica, hoy en día las aulas siguen siendo, en general, el marco donde se desarrolla casi en exclusiva la vida escolar, con algunas excepciones. Se debe salir de ese recinto para que el alumnado esté en contacto con el mundo que le rodea, para que no exista una dicotomía entre el aula y la vida real o, como apunta Vilarrasa (2003b, p. 15), una escisión entre lo aprendido ("el concepto") y la realidad ("su contexto"). En esta misma línea, Leoz (2011) se refiere al barrio como el espacio más inmediato de conocimiento para niños y niñas, y que se debería tener más en cuenta desde el ámbito escolar. No obstante, hoy en día parece tener poca importancia, en la formación del futuro profesorado, las actividades fuera del aula y la utilidad del medio como recurso didáctico, a tenor de las escasas salidas que realizan durante su proceso formativo.

Este trabajo pretende fomentar los aspectos señalados anteriormente; salir del aula para contextualizar los conceptos abordados en ella (anterior o posteriormente), y mostrarlo como un recurso didáctico en la formación del futuro profesorado. Fomentar entre nuestro alumnado la curiosidad, la capacidad de investigación, potenciar el cuidado y el respeto por el patrimonio y, en definitiva, hacerle consciente de las posibilidades que ofrece la ciudad como un gran aula de la vida. Como indica Vilarrasa (2003b, p. 24) "en la sociedad postindustrial salir del aula representa la oportunidad de devolver al estudiante el contexto en el que la información adquiere su sentido y puede ser transformada en conocimiento al servicio de proyectos personales y autónomos". Por eso, nuestro objetivo es que aprendan a reconocer el contexto en el que se encuentran, que se habitúen a mirar a su alrededor, detectando y analizando los elementos de su entorno desde un punto de vista tanto social como natural, conozcan su pasado, interpreten el presente y actúen en la exposición de sus conocimientos.

\section{El itinerario didáctico en entornos urbanos}

Entre las principales herramientas pedagógicas a utilizar cuando se pretende salir del aula, están las visitas guiadas $\mathrm{y}$, sobre todo, los itinerarios didácticos; muy enriquecedores cuando se trata de lugares urbanos (Valverde, Sequeiros y Loma, 2001; Martínez y García, 2008; Santolaria, 2014).

Mientras que la visita didáctica se da sobre un elemento determinado (que puede ser un museo, un castillo, una iglesia, etc.), el itinerario constituye un recorrido preestablecido que cuenta con diferentes paradas, y que puede realizarse en un entorno natural y/o urbano. Este recurso presenta un gran potencial integrador, transversal e interdisciplinar de las diferentes materias curriculares que integran el proceso educativo. Si la escuela debe preparar al alumnado para una vida activa en la sociedad en la que este debe insertarse, el itinerario didáctico, que permite observar aspectos reales del espacio, guiados de 
la mano de un docente, constituye una herramienta fundamental en la tarea educativa.

Insa (2002; s/p) lo define "como un recorrido o camino a seguir con diferentes puntos de parada o interés en determinados elementos de valor patrimonial o cultural". López y Segura (2013, p. 16) resaltan las cualidades educativas de este recurso y su integración legislativa de manera amplia, al indicar que "puede convertirse en un instrumento integrador y vertebrador del currículo, motivador y muy útil para trabajar diferentes competencias básicas, así como para introducir, desarrollar y complementar una gran variedad de objetivos y contenidos".

Hoy en día, las nuevas tecnologías (sistemas de información geográfica, visores cartográficos, los modelos abstractos) permiten su utilización por parte del profesorado, para que el alumnado obtenga un acercamiento y/o grado de conocimiento amplio de diversos y variados aspectos (Rubio, 2012). Sin embargo, aunque la implementación de las técnicas de la información y la comunicación con carácter espacial está cada día más presente en las aulas, parece necesario fomentar y tener en cuenta los recursos que ofrece el medio cercano, fuera de los límites del aula, y plantear diferentes itinerarios para obtener un contacto directo con el espacio, conocer los principales caracteres, fomentar las relaciones con el lugar y mejorar la sensibilidad hacia la conservación, el respeto y la puesta en valor de los hechos y acontecimientos natu- rales, históricos y patrimoniales; locales, cercanos y con una gran capacidad interdisciplinar.

Además, permite una gran carga de motivación; de realizarse adecuadamente, contribuye a mejorar el interés del alumnado por los elementos a enseñar, sobre todo si se hace partícipe a alumnas y alumnos. Permite tomar un contacto directo con aspectos teóricos que suelen verse únicamente en clase y que, en muchas ocasiones, no se aprehenden con facilidad si no se observan directamente (Olave, 2005). Además, puede servir para poner en práctica técnicas de observación y estudio del espacio. Tomando como referencia a Sánchez (1995), Olave (2005, p. 199) señala aspectos tales como un análisis por comparación de elementos y utilizar un marco único para el desarrollo de las destrezas cartográficas, así como despertar la inquietud ambiental, su cuidado y respeto y fomentar la motivación: "Los itinerarios aplicados al aprendizaje permiten prestar atención en su recorrido de acuerdo al diseño de diferentes aspectos de una realidad, que muchas veces en el trayecto habitual no se fija ni se observa, por lo tanto, no se conoce".

\section{La interdisciplinariedad y} posibilidades competenciales entre la didáctica de las Ciencias Sociales y Ciencias Experimentales en la formación del profesorado de Educación Primaria

Interdisciplinariedad 
Si atendemos a la legislación, tanto la recientemente derogada Ley Orgánica de Educación (LOE) como la nueva Ley Orgánica para la Mejora de la Calidad Educativa (LOMCE), establece como uno de los elementos clave del currículo las competencias, ocho en el caso de la anterior ley, siete en la nueva. La Organización para la Cooperación y el Desarrollo Económicos (OCDE, 2001) define competencia como "la capacidad de responder a demandas complejas y llevar a cabo tareas diversas de forma adecuada". Manifestado de otro modo, se podría decir que las competencias engloban tres saberes; saber ser, saber hacer y saber conocer, y han sido objeto de estudio de diferentes autores (Moya 2008; Méndez-Giménez 2013).

Por otro lado, volviendo a la nueva legislación LOMCE, en lo que respecta a la etapa primaria (RD 126/2014), cabe destacar la escisión de la antigua asignatura LOE de "Conocimiento del medio natural, social y cultural" en las materias de Ciencias Sociales, por un lado, y Ciencias Naturales, por otro. No obstante esta separación, trabajar ambas disciplinas a la vez puede resultar realmente beneficioso. Algunos autores como Cortés de Arabia (2007) han defendido que la interdisciplinariedad (combinar varias disciplinas con el objetivo de conseguir una mejora en el proceso de enseñanza aprendizaje) puede ser verdaderamente útil a la hora de conseguir el triple objetivo mencionado con anterioridad: saber, saber hacer y saber ser. Podríamos decir, en consecuencia, que la interdisciplina- riedad conlleva una relación mutua y complementaria entre disciplinas, en torno a un determinado objeto, fenómeno o hecho. En este trabajo proponemos como ese objeto un poderoso recurso pedagógico: el itinerario didáctico. Es más que evidente la riqueza y potencialidad del recurso ante el que nos encontramos, y aunque existen numerosos trabajos al respecto, pocos son los que trabajan conjuntamente conocimientos relacionados con las Ciencias Sociales y las Ciencias Experimentales en el mismo itinerario (Abril et al, 2008).

\section{Competencias desarrolladas}

Atendiendo a las competencias anteriormente mencionadas, resultan más que evidentes las posibilidades que este recurso ofrece:

Competencia en comunicación lingüística. El fomento de esta competencia es claro, ya que los distintos grupos, además de preparar y llevar a cabo una exposición para elaborar cada punto del itinerario, debían elaborar un dossier sobre éste.

Competencia matemática y competencias básicas en ciencia y tecnología. Fundamentalmente se fomentan las segundas, ya que cada grupo debía preparar dos puntos del itinerario, uno de ellos con algún tema relacionado con las ciencias experimentales y/o la tecnología, como el vehículo eléctrico o la locomotora de vapor.

Competencia digital. En una actividad como ésta, en la que los alumnos y alumnas deben buscar información 
sobre diferentes aspectos indicados por el profesorado, resulta fundamental la búsqueda de ésta en internet, además de la bibliografía recomendada.

Aprender a aprender. De manera complementaria a la anterior competencia, nuestro alumnado debe buscar información sobre aspectos propuestos por el profesorado y aprender a discernir la que es útil de la que no lo es. Además deben sintetizar todo aquello que encuentran, pues el tiempo dado para su exposición es limitado.

Competencias sociales y cívicas. En una actividad como ésta, realizada en grupos pequeños de entre tres y cinco estudiantes, resulta fundamental la coordinación y armonía entre sus integrantes a la hora de llevar a cabo un buen trabajo. Por otro lado, durante el itinerario didáctico es indispensable el respeto y silencio hacia quienes llevan a cabo sus exposiciones en cada punto del itinerario. Del mismo modo, a través del itinerario se favorece el vínculo del alumnado con su ciudad, mediante la integración de actividades didácticas en el entorno urbano.

Conciencia y expresiones culturales. De manera análoga a como sucede con las Ciencias Experimentales, la segunda disciplina puesta en alza en esta actividad son las Ciencias Sociales, ya que todos los grupos deben trabajar un tema relacionado con esta disciplina, bien Geografía, Historia o Historia del Arte. Además, mediante esta actividad se potencia el conocimiento del patrimonio y, por tanto, se fomenta el respeto por el mismo.

\section{Metodología de trabajo implementada en el proyecto}

En el itinerario que aquí se plantea, teniendo en cuenta el nivel universitario del alumnado al que va dirigido (dos grupos de clase, pertenecientes al Grado de Educación Primaria en el tercer curso de la Universidad de Córdoba, es decir, en torno a 120 estudiantes), implementa una metodología que ha partido de la base de la integración y la interdisciplinariedad entre dos asignaturas, en principio dispares, como son la Didáctica de las Ciencias Sociales y de las Ciencias Experimentales. Además, se tiene en cuenta que son los discentes, por grupos de cuatro o cinco, los encargados de preparar la exposición de las paradas, previamente establecidas por el profesorado y donde deben abordar unos objetivos establecidos por estos. Así, aprovechando el medio cercano, la ciudad de Córdoba, su entorno urbano y el imponente legado histórico y patrimonial, se les ha pedido abordar aspectos relacionados con la historia (la evolución histórica, la huella de las distintas culturas, las manifestaciones de las diversas sociedades, etc.), contenidos de geografía urbana que caracterizan la parada seleccionada (antiguos espacios industriales, planificación del tranvía, reutilización del patrimonio, nuevas áreas residenciales, etc.), así como cuestiones relacionadas con las ciencias experimentales (los medios de transporte y locomoción; el funcionamiento de los hornos de fábricas; el uso del agua desde un punto de vista tec- 
nológico, etc.), que en un futuro pueden servirles en las prácticas en sus clases de Educación Primaria. De este modo, el profesorado ha ejercido de guía a la hora de plantear las paradas propuestas y los objetivos a cumplir en cada una de las mismas por los diferentes grupos.

Ha sido tarea del alumnado preparar las paradas, buscar la información relativa a los elementos más importantes de las respectivas paradas, así como la exposición in situ de las peculiaridades del mismo en el punto de interés. El resultado ha sido un itinerario con siete paradas en ubicaciones emblemáticas de la ciudad. (En nuestro caso, la predisposición de las paradas responde al número de alumnos matriculados en la asignatura, lo cual obliga a crear una media de catorce grupos de trabajo, -4 o 5 integrantes- repartidos en dos jornadas de trabajo). No obstante, como señala Insa (2002) es recomendable reducir el número de paradas, buscar ubicaciones con poco tráfico y que estén relativamente cercanas.

Previamente al desarrollo de estas actividades, se había trabajado en clase el aparato teórico del desarrollo de un itinerario didáctico; sus principales funciones (como recurso motivador, integrador, evaluador, etc.), además de tener en cuenta los momentos en los que se puede dividir el itinerario (antes, durante y después), así como las diferentes metodologías que se pueden llevar a cabo en su desarrollo.

Cuando el itinerario es urbano, como es el caso, se debe decidir si se va a rea- lizar un recorrido diacrónico o, por el contrario, que sea un tema determinado el que actué como eje vertebrador del recorrido. En este caso, el profesorado ha omitido estos dos preceptos en los puntos de parada y se han buscado espacios y elementos donde se pudiesen realizar exposiciones en torno a las cuestiones relacionadas con las ciencias sociales y las ciencias experimentales, en cierto punto inconexas (La Torre del Chimeneón en el barrio de Ollerías; Plaza Parque de Colón; antigua estación de tren en la Avenida de América; Mausoleo romano en el paseo de La Victoria; antigua puerta de Almodóvar; alrededores de la Mezquita-Catedral; ribera del río Guadalquivir a la altura del puente romano).

En todo el proyecto ha tenido gran importancia el papel de la tutorización del profesorado; como herramienta a la hora de evaluar la participación de los estudiantes, involucrados en la preparación de la salida, y para solventar posibles errores de enfoque y solapamientos a la hora de tratar algunos contenidos. En este proceso, se ha podido comprobar el grado de autonomía y colaboración de los diferentes grupos entre ellos, así como la intervención de cada individuo en el mismo.

En la figura I se comenta brevemente la planificación realizada por parte del profesorado implicado, a la hora de desarrollar correctamente el itinerario. De esta forma, los primeros pasos fueron exponer, en una sesión teórica, la actividad-itinerario con las correspondientes paradas y configurar los grupos 


\section{Figura I \\ Planteamiento metodológico y temporalización de la actividad}

Fuente: Elaboración propia

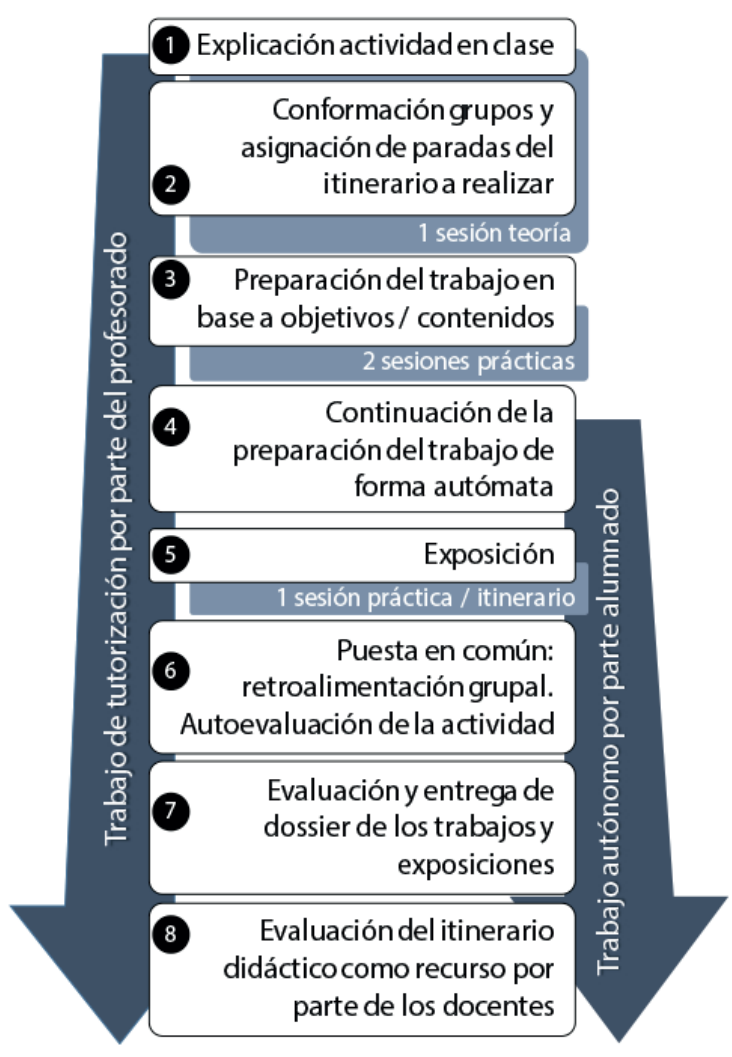

de trabajo. La preparación de las paradas asignadas a cada grupo conllevó dos sesiones prácticas, donde se abordaron desde la descripción de la parada hasta las referencias bibliográficas citadas en el dossier final, pasando por la realización de mapas conceptuales, esquemas y demás recursos infográficos. A partir de esta sesión, y hasta la salida y realización del itinerario, cada grupo de trabajo preparó un dossier-memoria, donde plasmaron los aspectos más relevantes de la parada seleccionada, así como el material a utilizar en el recorrido. Al concluir el itinerario, se abre un intercambio de ideas, tanto de aspectos didácticos como de los contenidos expuestos, intentando fortalecer las cuestiones trabajadas y dar solución a posibles errores. La evaluación final, tanto de las memorias como del potencial recurso didáctico, supuso la última fase de la planificación. Para finalizar, se debe indicar que todo el proceso debe estar convenientemente tutorizado por parte del profesorado responsable, lo que repercutirá en el buen resultado final de la actividad.

\section{Las potencialidades didácticas de la ciudad de Córdoba}

En relación con la cultura de masas de la etapa postindustrial, en las últimas décadas se han desarrollado iniciativas en diversos núcleos urbanos que buscan aprovechar su patrimonio histórico-cultural y natural desde un punto de vista educativo, orientado tanto al ámbito escolar como al público en general, a través del turismo (Hernández, 2002, p. 251-254). En este sentido, la ciudad de Córdoba se presenta como un enclave privilegiado para desarrollar itinerarios didácticos. La que fuera capital de la Bética romana y del Califato omeya, declarada Patrimonio de la Humanidad en 1994 por la Unesco, cuenta con un valioso legado histórico y monumental que facilita un acercamiento desde diversas perspectivas. 
Figura II.

Itinerario por la ciudad de Córdoba. Paradas: 1) Torre del Chimeneón;

2) Plaza Parque Colón; 3) Antigua estación de tren; 4) Mausoleo Romano;

5) Puerta de Almodóvar; 6) Alrededores de la Mezquita-Catedral;

7) Sotos de la Albolafia

Fuente: Elaboración propia sobre Google Maps

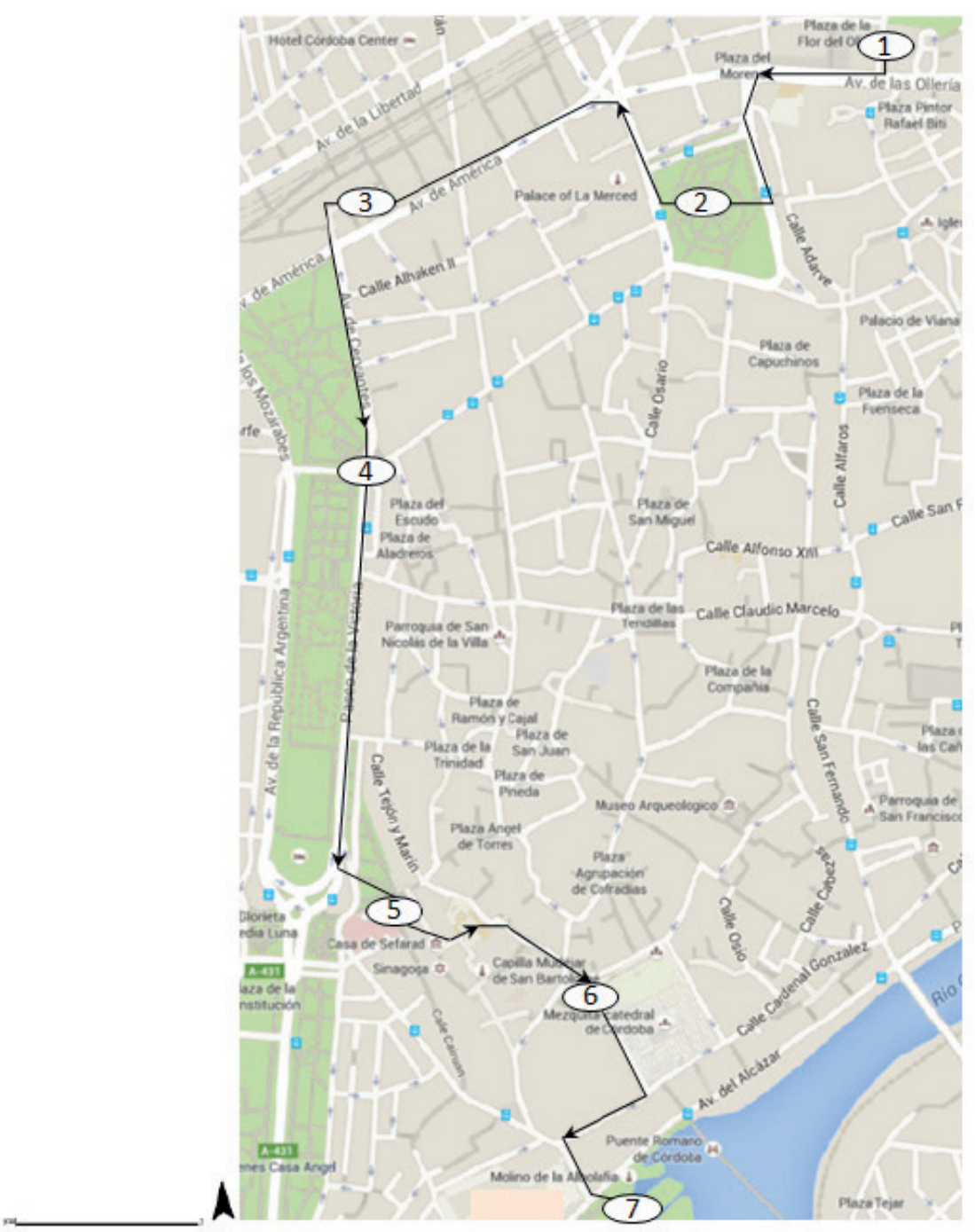

Precisamente, uno de los objetivos del itinerario es que el alumnado sea consciente de ese sincretismo, potenciando el valor de la interculturalidad y el cosmopolitismo. Acercarse a las sociedades del pasado permite desarrollar un sentimiento de empatía por las mismas y entender la relatividad de 
los valores propios de cada cultura, lo que redunda en la competencia social y ciudadana de nuestro alumnado (Santisteban y Pagés, 2007). Junto a ello, esta actividad también favorece que los y las estudiantes se interroguen sobre lo que están viendo y se familiaricen con las explicaciones multicausales de interrelaciones complejas; es preciso que entiendan el hecho de que, si bien compartimentamos la realidad para analizarla, tal división responde a un acuerdo científico y académico.

En ese sentido, hemos entendido el concepto de patrimonio en un sentido amplio, porque, junto a los elementos más significativos, como la Mezquita, nos hemos detenido también en enclaves menos conocidos (por ejemplo, una chimenea industrial), pero que son igualmente imprescindibles, siempre buscando la posibilidad de hablar de aspectos sociales (historia, arte, geografía, orientación, mapas...) y naturales (composición química, ingenios y maquinaria, flora y fauna...). De esta forma, nuestra pretensión era hacerles reflexionar sobre el entorno en el que se mueven a diario, para que fueran conscientes de ese legado y de las posibilidades didácticas de la ciudad.

El itinerario realizado consiste en la exposición y visita de siete enclaves urbanos de la ciudad de Córdoba, con la finalidad de reforzar los conocimientos teórico-prácticos de las asignaturas de Didáctica de las Ciencias Sociales y Didáctica de las Ciencias Experimentales. En la figura 2 se muestra el plano de la ciudad y el itinerario propuesto con las paradas realizadas durante el mismo.

\section{Parada 1. Torre del Chimeneón (Av. de} Ollerías)

El Chimeneón es una chimenea de la antigua fábrica San Antonio, perteneciente a la familia Carbonell. Esta fábrica de aceites fue construida en el año 1903, a las afueras de la ciudad y marcó la primera expansión industrial de Córdoba. Actualmente, ya en desuso, queda enmarcada dentro de la Plaza del Olivo y en su base hay una placa conmemorativa que habla de la industrialización de la capital y por ende, de la provincia.

El contenido de esta parada, por tanto, incide en esta industrialización. De esta forma, el grupo correspondiente debe preparar y exponer el proceso de fabricación del ladrillo y el funcionamiento de los hornos de fundición (referente al temario de Experimentales). A la vez se han de contemplar los factores de localización de esta antigua factoría, situada a las afueras de la ciudad a principios del siglo XX y cómo ha quedado integrada en ésta con el desarrollo de los nuevos ensanches. Constituye un símbolo del proceso fabril de la ciudad, y su protección patrimonial en la actualidad.

\section{Parada 2. Plaza Parque Colón}

Esta céntrica plaza de la ciudad recibe dos denominaciones. Se la conoce como Jardines de la Merced, debido a que en las proximidades se encuen- 
tra el antiguo Convento de la Merced (actual sede de la Diputación Provincial de Córdoba), donde todavía se conserva, tras severas restauraciones, la iglesia. También es conocida como Jardines de Colón, ya que está datado que en 1486 Cristóbal Colón residió, como invitado, en el convento señalado mientras esperaba audiencia con los Reyes Católicos.

Además de estas denominaciones, el emplazamiento también tiene una historia peculiar: habiéndose ubicado en esta zona una necrópolis romana, el parque fue, hasta 1831, plaza de toros, el Coso del Campo de la Merced y, desde 1835 hasta 1994, recibió numerosas remodelaciones para convertirse en la actual plaza ajardinada. Otra característica particular es que posee una mezquita (Morabito) que, tras acuerdo con el Ayuntamiento de la ciudad en 1992, está regentada por la Asociación Musulmana de Córdoba, siendo lugar de culto y reunión para esta comunidad.

En esta parada, los alumnos deben preparar y exponer la funcionalidad de la automoción en los tranvías y su uso como transporte urbano limpio (referente al temario de Experimentales) y la función social del tranvía, así como el contexto histórico de la plaza (referente al temario de Sociales). Este contenido se debe a que en el año 2004 hubo un proyecto en la ciudad, desechado con posterioridad por problemas de costes principalmente, para incluir al tranvía entre los medios de locomoción urbanos. Es destacable en esta parada la dicotomía que surge con la proyección del transporte urbano de carácter público, y su impacto tanto visual como económico.

Parada 3. Antigua estación de tren (Avenida de América)

La llegada del tren a la ciudad de Córdoba, fechado en 1859 y procedente de Sevilla, supuso la revitalización de la ciudad y una expansión demográfica y territorial cercana a la que posee en la actualidad. La antigua estación se hallaba, en esos primeros años, extramuros al noroeste de la ciudad y cercana a los Jardines de la Agricultura. Esta ubicación dio nombre a la estación, llamándose en primer lugar, Estación de la Agricultura y con el paso de los años, Estación Central.

El aumento demográfico de la ciudad, con el consiguiente incremento del número de personas viajeras, y la necesidad de soterrar las vías del tren, que dividían la ciudad en dos mitades, con un reparto desigual de la riqueza, conlleva a una reubicación de la estación en su actual ubicación, la Glorieta de las tres culturas.

La antigua estación se usa actualmente como la sede en Córdoba de la Radio Televisión de Andalucía y su función inicial está presente en una reproducción de un ferrocarril de caldera ubicado a la entrada de dicho edificio (figura III). En este punto, el alumnado tiene que preparar y exponer el tren como medio de transporte y su evolución técnica, desde los trenes de caldera hasta los de levitación magnética (en la parte de Experimentales) y, por otro 


\section{Figura III}

\section{Estudiantes exponiendo delante del ferrocarril de la antigua Estación}

Fuente: Elaboración propia

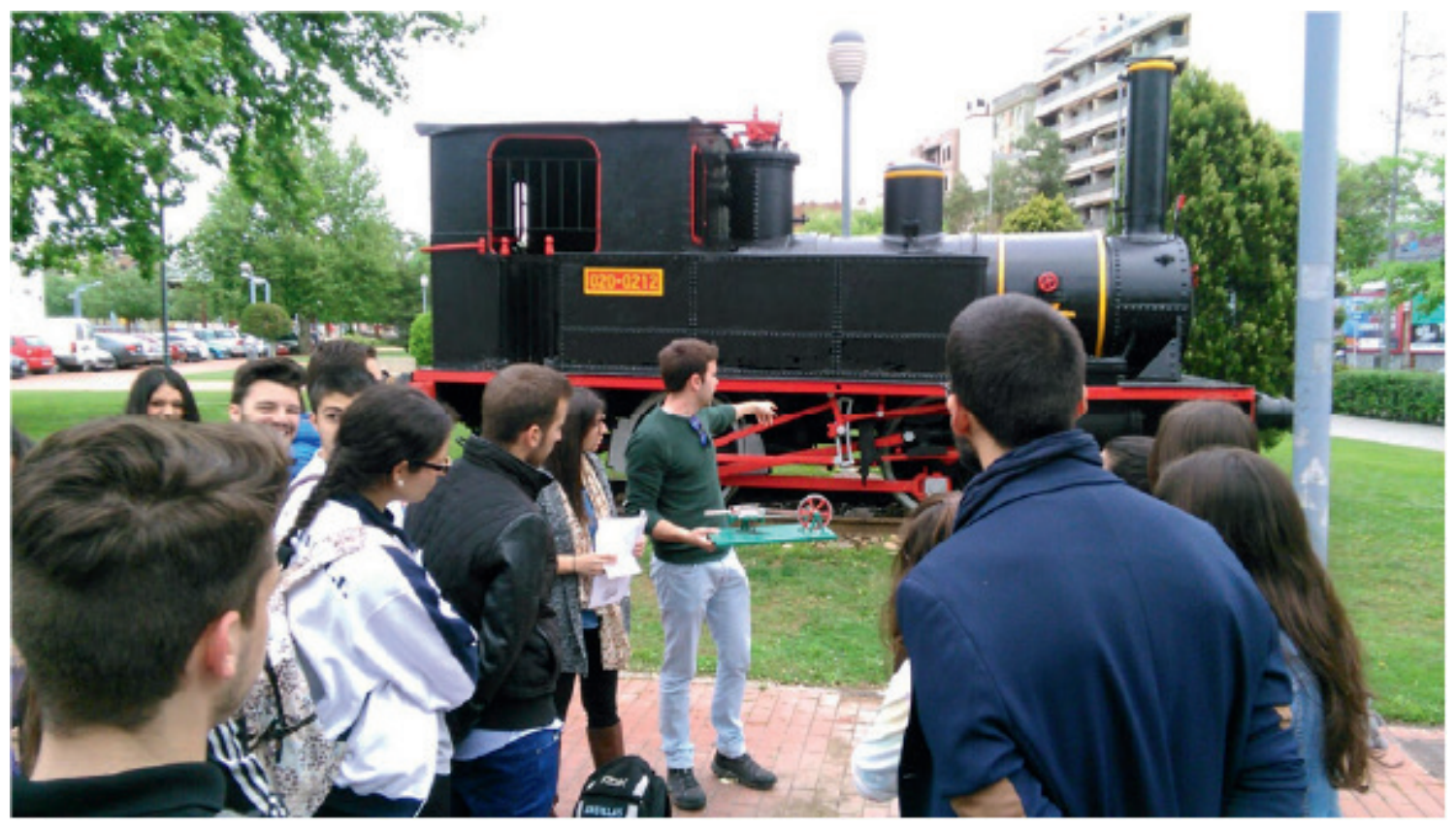

lado, el desarrollo del tren en la vertebración del territorio y su relación con el proceso de industrialización, de igual forma que su protagonismo en la comunicación de la ciudad de Córdoba con respecto al resto de España (en la parte de Sociales). En este sentido, cobra importancia entender los factores geográficos que sitúan la ciudad de Córdoba como nexo de caminos entre la meseta y la costa sur española; de ahí la presencia histórica de la red ferroviaria.

También, aprovechando el lugar y la temática planteada, es un punto apropiado para debatir someramente la funcionalidad de los diferentes medios de transporte utilizados en España, ventajas, inconvenientes, etc., y más concretamente ceñidos al transporte ferroviario.

Parada 4. Mausoleo Romano (Jardines de la Victoria)

Situado, en sus tiempos, a las afueras de ciudad y próximo a la puerta occidental de la muralla que circundaba el perímetro urbano de la misma, este monumento funerario fue descubierto en 1993 durante una excavación destinada a estudiar la viabilidad de un aparcamiento subterráneo. Debió ser construido en el siglo I d.C y por sus 
dimensiones y similitud con el panteón de Octavio Augusto, se cree que pertenecía a una familia acomodada. Este conjunto monumental consta de dos edificios (columbarios) homólogos, de los cuales sólo se conserva parcialmente, tras una restauración, uno de ellos.

En la excavación realizada, además de los columbarios, se encontraron restos de la vía romana que unía Córdoba con Hispalis (Sevilla) y actualmente es uno de los referentes arquitectónicos del pasado histórico de la ciudad en el imperio romano.

En esta parada del itinerario (figura IV), el grupo debe preparar y exponer cómo se fabricaban los ladrillos y la argamasa en la época romana, además de la tecnología de la época destinada a la construcción (referente al temario de la asignatura de Experimentales). Por otro lado, las principales referencias del legado romano en la ciudad de Córdoba, como el característico entramado urbano en damero y su relación con la estructura actual, lo cual puede también contribuir a apreciar aspectos arquitectónicos y urbanos. Igualmente, la funcionalidad del patrimonio, puesta en valor y medidas de conservación, orientado en la actualidad como recurso del turismo cultural.

\section{Estudiantes exponiendo en los restos patrimoniales del Mausoleo Romano de Córdoba}

Fuente: Elaboración propia

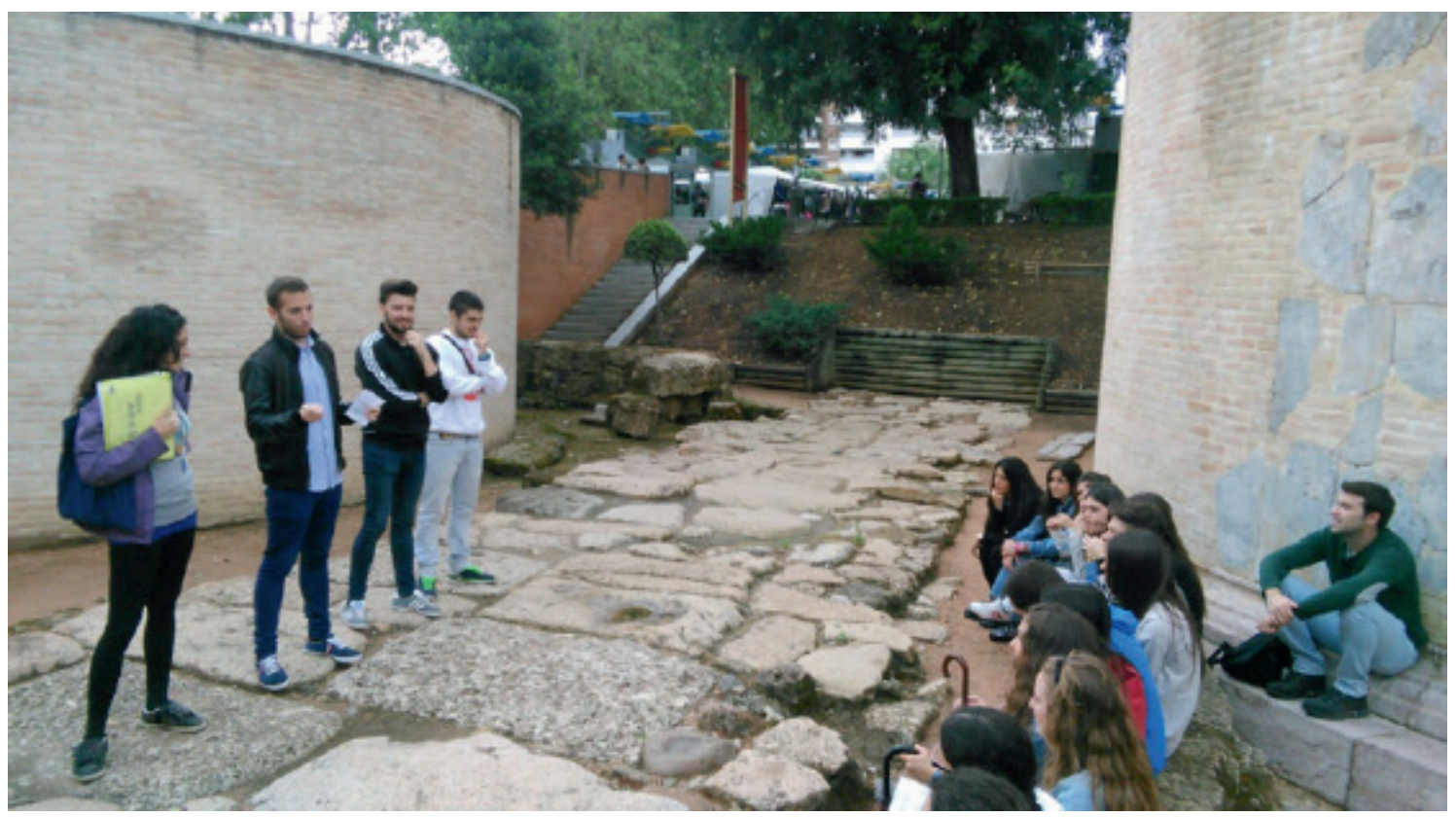


Parada 5. Puerta de Almodóvar (Calle Puerta de Almodóvar - Calle Cairuán)

El tratamiento del patrimonio también obedece a la siguiente parada; en este caso la puerta de Almodóvar. Constituye una de las entradas que se conservan del recinto amurallado de la ciudad de Córdoba. Situada en la parte oeste de la muralla, también se la conoce como puerta del Nogal o puerta de Badajoz (debido a encontrarse en el antiguo camino que unía ambas ciudades). De origen árabe, cerca de esta puerta hay una estatua dedicada al insigne filósofo y escritor cordobés Séneca. En tiempos de la Córdoba musulmana constituía un enclave particular que daba paso a la comunidad judía (testigo de ello son la Sinagoga y el remodelado Zoco - situados ambos en la actual calle Judíos) y por otro lado, a la zona noble de la ciudad, coronada por la Mezquita Grande (actual MezquitaCatedral).

El alumnado expone cerca de una fuente-aljibe, que recorre paralelamente la muralla desde esta puerta hasta la cercana Puerta de la Luna. En esta parada, tienen que preparar y exponer la importancia del agua y su uso como elemento indispensable para la vida, además de los sistemas de irrigación de tuberías, canalizaciones, acueductos, etc...., como ejemplos de ingenios técnicos de la antigüedad (tocando contenidos del temario de ambas asignaturas). También es importante considerar la presencia de este recurso en otros luga- res, así como los conflictos que genera su escasez.

Parada 6. Alrededores de la MezquitaCatedral (Calle Cardenal Herrrero)

La Mezquita-Catedral constituye, sin duda alguna, el conjunto arquitectónico más importante de la ciudad. Asentada sobre los restos de la iglesia visigoda de San Vicente Mártir, la mezquita fue construida en cuatro etapas: Abderramán I (780), Abderramán II (852), Alhakken II (960) y Almanzor (987). A partir de la toma de la ciudad por parte de las tropas del rey Fernando III en 1236 y el asentamiento de la cultura cristiana, se empieza a fraguar la idea de su conversión en catedral, terminándose esta obra-remodelación en 1569 por parte del insigne arquitecto Hernán Ruiz II.

En este punto del itinerario, el alumnado trabaja los tipos de materiales de construcción usados para hacer la mezquita, además de la situación de estas canteras de material (referente al temario de la asignatura de Experimentales). El hecho crucial es destacar que dichas canteras tienen un origen marino, lo que da sentido al descubrimiento de restos fósiles (bivalvos) en las rocas de la pared exterior del monumento. Con respecto al temario de la asignatura de Sociales, el alumnado prepara una disertación sobre la organización y distribución social en la Córdoba árabe y las diferencias culturales con respecto a las otras dos culturas de la ciudad (cristiana y judía). También es impor- 
tante considerar en esta parada la relación que presenta este monumento y su influencia en el turismo actual de la ciudad y, sobre todo, el importante papel económico que genera dentro del sector servicios.

\section{Parada 7. Sotos de la Albolafia (Avenida del Alcázar)}

Los sotos de la Albolafia constituyen un paraje natural de gran riqueza a nivel de biodiversidad, gracias, fundamentalmente, a la continua irrigación del rio Guadalquivir. En la época árabe esta zona constituyó un vergel donde proliferaron gran cantidad de cultivos, sobre todo viñedos y cereales. Esta revolución agrícola dependía claramente de una revolución tecnológica que evolucionó la forma de trabajar la tierra y de aprovechar los recursos naturales. De esta forma, el río constituyó un poderoso recurso, usado no sólo para los cultivos, sino para la ornamentación de fuentes y baños, además de su aprovechamiento para el consumo humano y animal. Esta revolución tecnológica también se refleja en molinos hidráulicos y en la noria (Albolafia), que da nombre a la parada del itinerario.

En funcionamiento hasta mediados del siglo XX, la noria servía para irrigar los jardines del actual alcázar. En esta parada, el alumnado debe hablar del funcionamiento técnico de la noria hidráulica y de ingenios similares que se aprovechan de la energía hidráulica (referente a la parte del temario de Experimentales) y del uso del uso del agua en la vida urbana de la ciudad, así como una reflexión sobre el papel del río Guadalquivir como referente histórico desde el punto de vista social. Además, también se debe considerar la función que tenían otros elementos anexos a la noria como los molinos hidráulicos. Por último, también es interesante reflexionar en esta parada sobre el cambio social de las ciudades, respecto a la apertura y adecentamiento de la ribera de los ríos, en contraposición al uso tradicional para la deposición de vertidos y actividades insalubres. El lugar ofrece, asimismo, elementos para trabajar desde el punto de vista patrimonial o de las ciencias naturales entre otras, debido a la riqueza de flora y fauna que se desarrolla en este paseo fluvial.

\section{Conclusiones}

En este trabajo se ha querido mostrar una metodología docente fruto de la colaboración entre dos áreas universitarias (Didáctica de las Ciencias Sociales y Didáctica de las Ciencias Experimentales) que comparten el mismo alumnado en el Grado de Educación Primaria, donde, como se ha visto, se ha puesto en marcha un trabajo teórico-práctico en relación al trabajo fuera del aula. El mismo ha consistido en la elaboración de un itinerario didáctico, donde el profesorado sólo ha señalado los puntos de parada en la ciudad de Córdoba y la temática en la cual se debían centrar; los y las alumnas, por su parte, en grupos de cuatro o cinco, han tenido que buscar y seleccionar la 
información, preparar los materiales y exponer in situ en una de las paradas seleccionadas. De este modo, a través de una metodología activa, el alumnado en formación participa en el proceso de elaboración de un itinerario, desarrollando competencias y capacidades que, en un futuro, deberá poner en funcionamiento.

Esta metodología permite fomentar la transversalidad y la cooperación entre diferentes áreas, en este caso integradas en el mismo departamento. El desarrollo de esta actividad ha requerido de la puesta en común de objetivos y contenidos, a través de varias reuniones de trabajo, que luego se han trasladado a las clases prácticas del alumnado, donde éstos han trabajado en la búsqueda de información y en la preparación de la exposición. Asimismo, la realización de un itinerario didáctico como éste permite trabajar distintas competencias, no sólo las más cercanas a las materias. Así, además de éstas, las competencias digitales y aprender a aprender, se fomentan con el trabajo de búsqueda de información por parte del alumnado. Por otro lado, las competencias lingüísticas y sociales y cívicas se potencian fundamentalmente durante las exposiciones del alumnado, en las que no sólo se pone énfasis en la manera de exponer, sino que también era tenido en cuenta el silencio, respeto e interés mostrado por el resto de compañeros y compañeras. Igualmente, competencias de carácter espacial, a la hora de localizar y realizar desplazamiento en el entramado urbano.
Aunque se está en proceso de análisis de unas encuestas de valoración y grado de satisfacción de la actividad, el grupo parece haber recibido con satisfacción la puesta en marcha de esta actividad. A través de este tipo de tareas, se permite a los y las estudiantes colaborar de forma más dinámica, e incidir en su motivación, al contrario de lo que sucede a menudo en las clases teóricas. Además, también pone en funcionamiento el interés y el análisis por cuestiones relacionadas con su entorno cercano, en este caso, en el ámbito de las cuestiones espaciales, sociales, arquitectónicas, culturales y patrimoniales que se dan en la ciudad de Córdoba. Este tipo de actividades permite al alumnado conocer su espacio más inmediato, peculiaridades y hechos que dan sentido a la ocupación humana del medio, en este caso de ámbito urbano. Se trata de un planteamiento extrapolable a otros ámbitos, adecuado no obstante a la casuística de cada espacio y contexto. Aunque esta experiencia docente está destinada a la formación del profesorado en el Grado de Educación Primaria, se puede plantear al propio alumnado de entre 9 y 12 años, con un enfoque participativo e integral.

\section{Referencias bibliográficas}

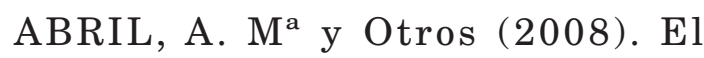
agua: itinerarios didácticos por el patrimonio histórico-artístico y espacios naturales de la zona de Sierra Mágina (Jaén). En Didáctica de las Ciencias Sociales, Currículo 
Escolar y Formación del Profesorado (703-704). Jaén: La casa del libro. Encuadernaciones.

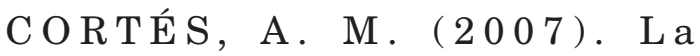
Interdisciplinaridad en la Educación Universitaria. Anuario del CIJS. Anuario $\mathrm{n}^{0} 10$, sección 4, 401-415. [En línea]. www.derecho.unc.edu.ar/ publicaciones/anuarios-del-cijs.../ anuario.../file. Fecha de consulta: 2 de febrero de 2016

HERNÁNDEZ, F. X. (2002). Sociedad, Patrimonio y Enseñanza. Estrategias para el siglo XXI. En GONZÁLEZ GALLEGO, I. (dir.), La Geografía y la Historia, elementos del medio (245-277). Madrid: Ministerio de Educación, Cultura y Deporte.

INSA, Y. (2002). Itinerarios urbanos, recursos y materiales didácticos para explicar la ciudad. Íber Didáctica de las ciencias sociales: Geografía e Historia, 32, 89-95

LÓPEZ, F.; SEGURA, J. (2013). Los itinerarios didácticos: un recurso interdisciplinar y vertebrador del currículum. Espiral, Cuadernos del Profesorado. Revista Digital del Centro de Profesorado Cuevas-Olula. Vol. 6, 12. Disponible en: http://www. cepcuevasolula.es/espiral.

MARTÍNEZ, F. J.; GARCÍA, A. J. (2008). Itinerarios didácticos por Fuente Álamo (Murcia), una estrategia educativa de innovación en el proceso de enseñanza aprendizaje en educación secundaria. Espiral Cuadernos del profesorado, 1, 1-9.
MARRÓN, M. J. (2005). Metodología lúdica y aprendizaje significativo en Geografía. Propuesta de un juego de simulación para la educación intercultural. Didáctica Geográfica, 7, 383-406.

- (2012). Aproximación al concepto de escala en el primer ciclo de Educación Primaria. Presentación de un juego para su tratamiento desde la enseñanza activa. Didáctica Geográfica, 13, 93-112.

MÉNDEZ,A., SIERRA, B. y MAÑANA, J. (2013). Percepciones y creencias de los docentes de Primaria del Principado de Asturias sobre las competencias básicas. Revista de Educación, 362. Septiembre-diciembre, 737-761.

MOYA, J. (2008). Las competencias básicas en el diseño y el desarrollo del currículum. Revista Qurriculum, 21, 57-78. [En línea]. Disponible en: http://revistaq.webs.ull.es/ ANTERIORES/numero21/moya.pdf. Fecha de consulta: 2 de febrero de 2016

OCDE (2001). Definition and selection of competencies: Theoretical and Conceptual Foundations (DeSeCo). 11 feb 2016, de OCDE Sitio web: http:// www.oecd.org/edu/skills-beyondschool/41529556.pdf

OLAVE, D. (2005). El itinerario didáctico: una propuesta metodológica para el análisis geo-histórico local. Geoenseñanza, 10, 197-208.

REYES, J. L., (2011). Didáctica de las Ciencias Sociales: vida cotidiana, 
conocimiento de sí mismo, y autonomía personal. En RIVERO GARCÍA, $\mathrm{M}^{\mathrm{a}}$. P. (coord.), Didáctica de las ciencias sociales para educación infantil (65-88). Zaragoza: Mira.

RUBIO, P. (2012). Itinerario didáctico por la Sierra de Albarracín. Serie Geográfica n 11, 101-116.

RUIZ, C.; PALACIO, I. (1999). Higienismo, educación ambiental y previsión escolar. Antecedentes y prácticas de Educación Social en España (1900-1936). Valencia: Universitat de València.

SÁNCHEZ, A. (1995). El trabajo de campo y las excursiones. Enseñar Geografía. De la teoría a la práctica. Síntesis. Madrid

SANTISTEBAN, A.; PAGÉS, J. (2007). La educación democrática de la ciudadanía. Una propuesta conceptual. En ÁVILA, R. M ${ }^{a}$; LÓPEZ, J. R.; FERNÁNDEZ, E. (eds.), Las competencias profesionales para la enseñanza- aprendizaje de las ciencias sociales ante el reto europeo y la globalización (353-368). Bilbao: AUPDCS.

SANTOLARIA, A. (2014). La ciudad como recurso didáctico. EDETANIA, $45,235-244$

VALVERDE, F.; SEQUEIROS, C. y LOMA, M. (2001). La ciudad y el patrimonio urbano como forjadores de identidad. En ESTEPA, FRIERA Y PIÑEIRO. Identidades y territorios: un reto para la didáctica de las Ciencias Sociales (579-586). Oviedo: KRK y AUPDCS

VILARRASA, A. (2003a). Salir del aula, Íber. Didáctica de las ciencias sociales, geografía e historia, 36, 5-6.

- (2003b). Salir del aula. Reapropiarse del contexto, Íber. Didáctica de las ciencias sociales, geografía e historia, $36,13-25$.

RD 126/2014, de 28 de febrero, por el que se establece el currículo básico de la Educación Primaria. 
